\title{
A new Kind of Operations Inventory: the Pre-assembled Kit
}

\author{
Claude Machline \\ Escola de Administração de Empresas de São Paulo, da Fundação Getulio Vargas \\ claude.machline@fgv.br
}

\begin{abstract}
This paper studies the effect of a kit system on the supplier inventory. Kits have been popular for decades in many segments, especially in the health care sector. Pre-assembled kits create new kinds of inventory: a circulating inventory and its safety stock. The classic replenishment formula of the periodic review system is affected by the use of kits. The paper establishes simple formulas to determine the size of the circulating inventory and of its safety stock. The theme was inspired by the activities of an importer-distributor of orthopedic surgical goods, based in São Paulo, Brazil, who imports these items from an American manufacturer and loans the kits to hospitals for a few days to perform surgeries. Working with pre-assembled kits offers many advantages: less errors, less losses, more speed, which offset the moderate inventory increase they cause.
\end{abstract}

KEY WORDS: Kits logistic, Pre-assembled kits, Surgical kits, Inventory Management, Circulation inventory

\section{INTRODUCTION}

\section{Objectives of the Paper}

For some decades, kits - an assembled assortment of goods, prepared beforehand - have been popular in some areas, especially in the health care segment. This paper addresses the following questions and provides the corresponding answers: are kits money savers? Mostly yes. Do kits reduce inventory? No, they increase inventory, but to a small extent. Are kits practical? Very much indeed. Do they increase the needs for more inventory control? Yes, they render inventory control more difficult. Do they improve traceability? No, they render it more difficult. Do kits improve health care quality? Definitely, they reduce errors. Do they improve the logistics? They do, they answer to the well known material handling consolidation principle, making transportation easier and reducing substantially the delivery time. The paper provides some formulas to quantify the findings. A specific case of kits used in orthopedic surgery, manufactured in USA and distributed in São Paulo hospitals, is taken as an illustrative example.

\section{AN OVERVIEW OF THE PAPER}

An extremely complex logistic operation is the delivery to hospitals of highly specialized orthopedic surgical devices - equipment, instruments and supplies -. Those materials, too expensive for most hospitals to acquire and carry, are frequently lent by the supplier - manufacturer or distributor -, who delivers them on the eve of the scheduled surgery and retrieves them one day after. A characteristic of orthopedic surgery is that the supplier must send a whole set of implants - prostheses, anchors, bolts and screws-, since there is no way for the surgeon to know beforehand the exact size of the piece that he will have to use in order to replace or fix the damaged joint or bone. During the operation, after the removal of the destroyed part, the surgeon will choose, from a wide assortment of pieces, the ones that best fit to the patient.

These circumstances compel us to create some new concepts, concerning inventories. There is, to start with, an inventory of surgical parts, live implants and prostheses, which go from the supplier to an hospital, then back to the supplier, then to another hospital. The specific parts that the surgeon has uti- 
lized are replaced by items taken from the inventory room of the supplier. If the supplier attends to forty surgeries per day, he will have forty sets of goods moving to hospitals and back from them. This mobile amount of items might be called a "circulation inventory". Like all productive inventories, this one will require a safety inventory, on account of emergency surgeries, those due, not to chronic diseases, but to accidents or to violence. Orthopedic surgeries exist of many kinds. Each part of the human body - spine, hip, shoulder, knee, arm, elbow, hands and feet, and other places - requires specific instruments and equipment for the surgery to be performed. These assets, worth tens of thousand dollars, are also put at the disposal of the hospitals for the duration of the surgery. At the present state of the art, this procedure, which requires much transportation, is mandatory.

Most suppliers provide kits for the process described above. For instance, the set of shoulder anchors consists of seven units corresponding to diverse sizes, from diminute, small and median to extralarge. Each anchor comes from the manufacturer in a little box, duly sterilized. A kit shall carry these seven sizes. Now, there exist three quality levels of anchors, according to the material used: steel, titanium, or calcium phosphate covered alloy. This chemical is bioabsorbed by the body, producing a better adherence. The prices are in the relationship of 1:2:2.5. As the surgeon does not know, when he schedules the surgery and orders the supplies, which quality the health plan (the payer, the "third party") will authorize, the distributor will send many times a kit with these 21 pieces. Only one anchor will be used and 20 will revert to the distributor. Screws, surgical thread, gloves, drugs and other supplies are also added in the kit. The idea is to put in the kit everything the surgeon will possibly need. Whenever prepared in advance, the kits create a new kind of inventory: the pre-assembled kit inventory. The kit might be prepared well before any shoulder surgery be scheduled. The inventory consists of loose anchors on the shelf and anchors secluded in kits, composing the "kits inventory". How many kits shall a distributor assemble? Shall he carry a "kit safety stock"? These questions are addressed next.

\section{KITS DEMAND AND SUPPLY}

Surgeries are not evenly distributed among the days of the week. For that particular distributor, surgeries are distributed in the way shown in the following table 1.
Table 1 - Distribution of surgeries among week days

\begin{tabular}{lcc}
\hline Week days & $\begin{array}{l}\text { Average of } \\
\text { daily shoulder } \\
\text { surgeries }\end{array}$ & $\begin{array}{c}\text { Average of daily } \\
\text { total surgeries }\end{array}$ \\
Monday & 6 & 15 \\
Tuesday & 6 & 15 \\
Wednesday & 8 & 20 \\
Thursday & 20 & 50 \\
Friday & 14 & 35 \\
Saturday-Sunday & 4 & 5 \\
\hline Total & 58 & 140 \\
\hline Daily average & $58: 7=9$ & $140: 7=20$ \\
\hline
\end{tabular}

The supplies, the instruments and the equipment must be sent at the hospital 24 hours before the surgery (day D-1); and must be removed from the hospital the day after the surgery (day D+1).

Therefore, once the surgery has been scheduled and the materials ordered, the whole set is committed for three days.

On Tuesday, for instance, the status of the circulating inventory is shown in the following table 2.

Table 2 - Tuesday Status of Circulation Inventory

\begin{tabular}{lc}
\cline { 2 - 2 } & No of sets \\
\hline $\begin{array}{l}\text { Being assembled for delivery to hospitals on } \\
\text { Being removed from hospitals after Monday } \\
\text { surgeries and available for a new cycle }\end{array}$ & 20 \\
$\begin{array}{l}\text { In surgery rooms at hospitals } \\
\text { Already delivered to hospitals for Wednesday } \\
\text { surgeries }\end{array}$ & 6 \\
Total circulating inventory & 8 \\
\hline
\end{tabular}

On Wednesday the circulating inventory status will look as follows:

Table 3 - Wednesday status of circulating inventory

\begin{tabular}{lc}
\cline { 2 - 2 } & № of sets \\
\hline $\begin{array}{l}\text { Being assembled for delivery to hospitals on } \\
\text { Wednesday for Friday surgeries }\end{array}$ & 14 \\
$\begin{array}{l}\text { Being removed from hospitals after Tuesday } \\
\text { surgeries and available for a new cycle }\end{array}$ & $(6)$ \\
$\begin{array}{l}\text { In surgery rooms at hospitals } \\
\text { Already delivered to hospitals for Thursday } \\
\text { surgeries }\end{array}$ & 8 \\
Total circulating inventory & 20 \\
\hline
\end{tabular}


Machline, C.: A New Kind of Operations Inventory: The Pre-assembled Kit

The circulating inventory status for each week day is found to be:

Monday: $20 \bullet$ Tuesday: 28

Wednesday: 36 - Thursday: 34

Friday: $28 \bullet$ Saturday-Sunday: 22

If the supplies are sent as a set, mounted whenever it leaves for an hospital, and dismounted and replaced in the shelves as units whenever they return from the hospital, the previous results indicate that the distributor should keep a minimum of 36 sets on the shelves.

Actually, on account of extended holidays; on medical conventions, which create, before and after, peaks of surgeries; and on delays in the return of the goods from the hospitals, the distributor should also carry some circulation safety stock, of, say, $10 \%$ of the circulation inventory. In our numerical example, this safety stock would amount to 4 sets. The total minimum inventory would be 40 sets.

But, if one uses kits, the supplies are not returned to the shelves as units, the circulation inventory will be 36 the whole week, a substantial amount of material will be tied in specific kits and be unavailable for other utilizations. Adding the safety stock of kits, the total increase in the inventory created by the use of a kit system would be 40 units, in the case given.

\section{Are Kits Money Savers?}

Permanent kits, of the kind described in this paper, save money in the following ways:

\section{Kits prevent errors}

When the supplies kit returns from an hospital the contents must be checked. The few materials which were used by the surgeon must be replaced. There is no need to put back the materials to the shelves, nor to mount the kit again, performing operations that are fertile sources of errors. The lack of an implement needed by a surgeon could be fatal to the patient and ruinous to the supplier. If one has to mount a set in an emergency it is easy to make a picking error, like confounding codes or mixing up some similar materials. Reliability, as a whole, is improved by a pre-assembled kit system.

\section{Kits save labor}

Permanent kits do not have to be dismounted and remounted, saving therefore much handling.

\section{Kits reduce losses and pilfering}

It is easier to notice the lack of a drug or of an instrument in a permanent kit than in a mere set of materials always arranged in a different order.

Kits increase the speed of logistics operation and save time

Supplies and instruments must be checked to see whether anything is lacking whenever delivered to an hospital and again, when returned to the distributor. Actually, on each cycle, these products are checked when they: 1) leave the distributor inventory room; 2) get to the hospital; 3) are received by technicians in the sterilization area and the operation room; 4) are removed from the operation room; 5) return to the supplier inventory. They are checked for physical, actual presence and for their matching with the invoice and bills that accompany them. One has to remove the whole content of the containers to perform an accurate check.

If the kit is prepared in advance and sealed, it is sufficient to check the nature and the number of kits handled, saving much time. In emergency situations, kits allow instant delivery, since there is no need for picking nor packing.

\section{Kits and Inventory Control}

Supplies utilized in the health care segment must comply which very stringent rules and controls, which render inventory control more difficult in this area than in most others. The inventory system must be able to offer lot (batch) control, so as to provide traceability, which means tracking all the way up to the source the origin of any problem; and to monitor the expiration date (validity date) of each input, which makes mandatory exhausting the previous batch before using the next one (FIFO - FIRST/IN/FIRST/OUT). As the kit constitutes an additional level of inventory, the distributor needs to possess an information system able to register all transactions involving each part that enters and leaves the kit.

\section{Some Formulas Involving a Kit System}

The demand for kits and the size of the circulating kit inventory were already treated in a previous part of this paper. One needs now to develop the replenishment formula for the purchase of the kit components; the formula for the safety inventory of 
the kit components and the formula for the safety inventory of the kit.

The replenishment system used by our São Paulo importer-distributor is of the periodic review kind. A demand forecast is made every month for the next two months for each part (stock keeping unit). We call $\mathrm{d}=9$ units this daily demand for a specific part; $\mathrm{R}=30$ days, the review period; $\mathrm{B}$, the safety inventory for this part; $\mathrm{L}=60$ days, the lead time for a part to be ordered, prepared in USA, packed, sent to Brazil, freed at the customs and be available on the importer - distributor shelves; EI, the existing inventory of this part on the day the purchase order is sent to the manufacturer; $\mathrm{PO}$, the pending orders, i.e., the purchase orders not yet delivered and available. $Q$ is the quantity to be ordered monthly for the part.

$$
\mathrm{Q}=\mathrm{d}(\mathrm{L}+\mathrm{R})+\mathrm{B}-\mathrm{EI}-\mathrm{PO}
$$

The classic formula (1) should be modified into (2) if the importer-distributor uses a kit system, to take into account the units tied in the circulating kits, which amount to the inventory $\mathrm{KCI}$, already computed, equal to $36 \mathrm{kits}$, and also the units tied in the safety kit circulating inventory SKCI, which was availed to be 4 kits, but which will be now computed more exactly. We assume that each kit carries only one unit of the focused part, and that this part goes only in that kit; otherwise, the next formulas will have to be modified, taking into account that $\underline{m}$ units go in each kit and the fact that this part is also used in $\underline{n}$ other kits. $\mathrm{Q}$ becomes:

$\mathrm{Q}=\mathrm{d}(\mathrm{L}+\mathrm{R})+\mathrm{B}+\mathrm{KCI}+\mathrm{SKCI}-\mathrm{EI}-\mathrm{PO}$

The classic formula for computing a safety inventory is the following (3). It assumes that demand and lead time are independent variables:

$$
B=\alpha \sqrt{ } \text { L. } \sigma_{d}^{2}+d^{2} \cdot \sigma_{L}^{2}
$$

In (3),

$\alpha$ is a factor which determines the safety level, and is usually taken as 1,96 or as 2 . This last value corresponds to a probability of more than $97.5 \%$ to have the parts demanded available in stock.

$\sigma_{d}$ is the standard deviation of the daily demand distribution

$\sigma_{L}$ is the standard deviation of the lead time distribution
Assuming $\alpha=2, L=60$ days, $\sigma_{d}=1$ unit, $d=9$ units, $\sigma_{\mathrm{L}}=15$ days,

formula (3) gives:

$$
B=2 \sqrt{ } 60 \times 1+9^{2} .15^{2}=270 \text { units, }
$$

which amounts to 30 demand days.

We proceed now to compute the exact value of the safety kit circulating inventory, SKCI. The formula to be used is ( $\left.3^{\prime}\right)$, similar to (3), but modified to take into consideration the maximum daily demand $\mathrm{k}=$ 20 kits, for kits; $\mathrm{L}=3$ days, the lead time for kits to close the cycle; $\sigma_{k}=2$ kits, the assumed standard deviation of the maximum daily demand distribution of kits; and $\sigma_{\mathrm{L}}=1$ day, the standard deviation of the kits lead time distribution. The classical formula (3) was developed for an unique supplier. In the present case, the suppliers are 20 hospitals, that return the kits. A small proportion $\beta=20 \%$, say, might delay the devolution of the kits.

$$
\text { SKCI }=\alpha \sqrt{ } \text { L. } \sigma_{k}^{2}+\beta^{2} \cdot k^{2} \cdot \sigma_{L}^{2}
$$

In our numerical example, (3') becomes:

$$
\mathrm{SKCI}=2 \sqrt{ } 3 \times 2^{2}+0,2^{2} \cdot 20^{2} \cdot 1=11 \text { kits }
$$

Computing $\mathrm{Q}$, from formula (2), one has:

$$
\mathrm{Q}=9(60+30)+270+36+11-\mathrm{EI}-\mathrm{PO}
$$

If demand is precisely equal to the forecast and if everything proceeds exactly as predicted, EI, the expected inventory, just after receiving the monthly batch, equal to a month consumption ( $9 \times 30=270)$, will be: $270+36+11+270=587$, and $\mathrm{PO}$, pending orders, will be 270 . Then $\mathrm{Q}$ will be equal to:

$\mathrm{Q}=9(60+30)+270+36+11-587-270=270$ units

The average inventory is:

$\mathrm{AI}=(9 \times 30) / 2+270+36+11=452$ units

The inventory increase attributed to the use of a kit system is: $36+11=47$ units, which is about $10 \%$ of the average inventory.

We conclude that the kit model causes a moderate increase in the inventory, of about $10 \%$, but that its advantages, in terms of quality, speed, and availability, more than offset this shortcoming. 


\section{The Special Kit}

It was assumed up to now that the part, an anchor, for instance, was always used in only one kind of kit. But it is usual that anchors, and many other items, be used in several kinds of kits. There are actually different kinds of shoulder surgeries, knee surgeries and spine surgeries. For instance, a shoulder anchor might be used in four different shoulder surgeries. If one uses a kit system, one has to prepare kits for four different uses. The supplier must stock four different types of kits instead of only one type. In that case, assuming that each of the four different surgeries has the same demand, the daily peak demand for any surgery becomes: $k^{\prime}=20 / 4=5$ units. Let us call $s$ the standard deviation of the daily peak demand for any shoulder surgery.

Since $\sigma^{2}{ }_{k}=4 s^{2}$, we have: $s=\sigma_{k} / \sqrt{ } 4=2 / 2=1$.

Formula ( $\left.3^{\prime}\right)$ becomes: SKCI' $=\alpha \sqrt{ } l \cdot \mathrm{s}^{2}+\beta^{2} \cdot \mathrm{k}^{\prime 2} \cdot \sigma^{2}=2 \sqrt{ }$ $3 \cdot 1+0,2^{2} \cdot 5^{2} \cdot 1=2 \sqrt{ } 3+1=2 \sqrt{ } 4=4$ units. Since there are four different kinds of kits, the buffer stock is 16 units. In comparison with the previous situation, in which there was only one kind of kit, which required a safety inventory of 11 kits, there is an increase of $16-11=5$ kits, an increase of $45 \%$ in the buffer inventory. Notice that this computation constitutes an application of the "square root law" of aggregate safety stock.

There is an aggravating consideration which one must face. We mentioned till now the existence of only one kind of anchor. But there are actually two sizes of anchors. Each one must be seen as a stock keeping unit. So, actually, there are not 9 daily average anchors used, but 4,5 of small ones and 4,5 of large ones. This circumstance is worsening still more the inventory increase caused by the use of kits.

The probability that special kits create some slow moving items cannot be overlooked. If the daily average demand for a "commodity kit" is nine (9) units, applying the Poisson distribution, the probability that there will be no demand is $e^{-9}=0,01 \%$. But if this demand is divided in four (4) different surgeries and four (4) different kinds of special kits, this probability becomes: $\mathrm{e}^{-2,25}=10,5 \%$ for any of the kinds of kits.

\section{CONCLUSION}

Orthopedic surgeries require very specialized and expensive equipment, instruments and supplies.
Many hospitals that perform these surgeries do not acquire these materials. They lend them from distributors. They are sent to the hospital on the eve of the surgery and return to the supplier the day after. This situation creates a new concept of inventory: the circulating inventory. In order to reduce logistics errors and attend more quickly to emergencies, distributors use pre-assembled kits. All the components necessary for a given kind of surgery are separated in advance and segregated in a large box, which constitutes the kit. As the demand is not deterministic and presents daily fluctuations, the commitment of materials to kits increases the circulating inventory and its respective safety stock. Some computations are done in the paper to show how to dimension the circulating inventory, its buffer and the periodic purchase formula. The advantages of the kit system - quality and speed - outweigh its disadvantages - moderate inventory increase, more difficulties in control and traceability.

\section{REFERENCES}

Bowersox, Donald J. and David J. Closs, Logistical Management, The Integrated Supply Chain Process, McGraw-Hill, NY, 1996.

Choobineh, Fred and Esmail Mohebbi, "Material planning for production kits under uncertainty", Production Planning and Control, Vol. 15, nº 1, Jannuary 2004, pp. 63-70.

Chopra, Sunil and Peter Meindl, Supply Chain Management Strategy, Planning and Operation, Prentice-Hall, Inc. Englewood Cliffs, N.J., 2001.

Croxton, Keely L. and Walter Zinn, "Inventory Considerations in Network Design" Journal of Business Logistics, Vol. 26, nํㅜ 1, 2005, pp. 149-168.

Hadley, G., and T. M. Whitin, Analysis of Inventory Systems, Prentice-Hall, Inc., Englewood Cliffs., N.J. 1963.

Ronen, B. "The complete kit concept", International Journal of Production Research, Vol. 30, nº 10, 1992, pp. 2457-2466.

Zinn, Walter, Levy, Michael and Bowersox, Donald J., "Measuring the Effect of Inventory Centralization/Decentralization on Aggregate Safety Stock: The "Square Root Law" Revisited", Journal of Business Logistics, Vol. 10, no 1, 1988, pp. 1-14.

\section{AUTHOR'S BIOGRAPHY}

Claude Machline is Emeritus Professor of the São Paulo Business School of Getulio Vargas Foundation. He graduated as chemical engineer at Rio de Janeiro Federal University; he is Master of Arts by the Michigan State University and obtained his Ph.D. degree at the Stanford University. 